\title{
Antidiabetic Bis-Maltolato-OxoVanadium(IV): Conversion of inactive trans- to bioactive cis-BMOV for possible binding to target PTP-IB
}

\author{
Thomas Scior ${ }^{1}$ \\ Hans-Georg Mack ${ }^{2}$ \\ José Antonio Guevara García ${ }^{3}$ \\ Wolfhard Koch ${ }^{4}$ \\ 'Departamento de Farmacia. \\ Facultad de Ciencias Químicas, \\ Benemérita Universidad Autónoma \\ de Puebla, Colonia San Manuel, \\ Puebla, Mexico; ${ }^{2}$ Institut für \\ Physikalische Chemie, Universität \\ Tübingen, Tübingen, Germany; \\ ${ }^{3}$ Laboratorio de Investigación en \\ Bioinorgánica y Biorremediación \\ (LIByB). Departamento de Ciencias \\ Básicas, Ingeniería y Tecnología, \\ Universidad Autónoma de Tlaxcala, \\ Apizaco, Tlaxcala, Mexico; ${ }^{4}$ Facultad de \\ Estudios Superiores Zaragoza (FESZ), \\ Universidad Nacional Autónoma de \\ México (UNAM), Colonia Ejército \\ de Oriente, Delegación Iztapalapa, \\ Mexico City, Mexico
}

\begin{abstract}
The postulated transition of Bis-Maltolato-OxoVanadium(IV) (BMOV) from its inactive trans- into its cis-aquo-BMOV isomeric form in solution was simulated by means of computational molecular modeling. The rotational barrier was calculated with DFT - B3LYP under a stepwise optimization protocol with STO-3G, 3-21G, 3-21G*, and 6-31G ab initio basis sets. Our computed results are consistent with reports on the putative molecular mechanism of BMOV triggering the insulin-like cellular response (insulin mimetic) as a potent inhibitor of the protein tyrosine phosphatase-1B (PTP-1B). Initially, trans-BMOV is present in its solid dosage form but in aqueous solution, and during oral administration, it is readily converted into a mixture of "open-type" and "closed-type" complexes of cis-aquo-BMOV under equilibrium conditions. However, in the same measure as the "closed-type" complex binds to the cytosolic PTP-1B, it disappears from solution, and the equilibrium shifts towards the "closed-type" species. In full accordance, the computed binding mode of cis-BMOV is energetically favored over sterically hindered trans-BMOV. In view of our earlier report on prodrug hypothesis of vanadium organic compounds the present results suggest that cis-BMOV is the bioactive species.
\end{abstract}

Keywords: vanadium compounds, diabetes mellitus, molecular modeling, computational quantum chemistry, PTP-1B

\section{Introduction}

Vanadium is actually known as a trace element, essential for higher organisms although deficiency symptoms in humans have not yet been clearly identified (Guevara 1996). Trapping free oxygen radicals that might cause cardiac problems, it acts as a biological antioxidant and shows antitumoral activity (Scior et al 2005). Bis-Maltolato-OxoVanadium(IV) (BMOV) is under investigation in other pathological processes induced by uric acid, such as vascular endothelial dysfunction (Jindal et al 2007).

The development of innovative vanadium compounds against diabetes mellitus (DM) has attracted much pharmaceutical interest during the last decades (Saatchi et al 2005). In the light of desired clinical advantages of a novel orally administered drug with selective insulin-like effects, a considerable impact on the patients' health, their insulin injection-related long-term complications, or reduced side effects compared to other oral antidiabetics, can be expected (Zhang et al 2008). Besides our review with critical details on the protein tyrosine phosphatase (PTPase) inhibition, the pharmacodynamical behavior of organic vanadium compounds, their complex stabilities and inconclusive role as prodrugs for simpler inorganic vanadium ions in combination with toxicity data, it is noteworthy here to add newer reports on pharmacological findings (Scior et al 2005). In 2007 the action mechanism on a molecular level was studied in BMOV-treated cell cultures. It was confirmed that BMOV enhanced the tyrosine-phosphorylation 
of upstream insulin-signaling proteins including the insulin receptor (Bose et al 2007). Heinemann and colleagues (2003) examined the pharmacokinetics of insulin mimetic inorganic vanadium ions: mainly vanadate (+V, 80\%) and other oxidation states. Their study in healthy humans after administration of a single intravenous dose revealed that the apparent volume of distribution was of the order of $0.6 \mathrm{~L} / \mathrm{Kg}$. A long terminal half-life dominates $(80 \%)$ the excretion phase. It is responsible for the typical heavy metal accumulation of vanadium in tissues such as liver, kidneys, bone, and spleen (Heinemann et al 2003). Most recently, another pharmacokinetics study in rats evaluated positively the therapeutical potential of BMOV as an oral glucose-lowering drug candidate. BMOV was rapidly absorbed, widely distributed in various tissues but only slowly eliminated from plasma due to accumulation to a greater extent in bone tissue. The intragastric bioavailability was around $25 \%$ of the initial doses. Significant decrease of glucose concentrations in plasma was observed in hyperglycemic diabetic rats (Zhang et al 2008).

Among the protein-tyrosine phosphatases, PTP1B has received much attention due to its role as a negative regulator of insulin signaling (Elchebly 1999; Cheng et al 2002). On one side the protein tyrosine phosphatase-1B (PTP-1B) is highly expressed in insulin-resistant persons. The absence of PTP-1B in genetically modified mice, on the other side, immunizes them against obesity and diabetes, even with rich and fatty diets. Clearly, a selective control of the biological function of PTP1B is a challenging task, and an inhibitor must not only efficiently bind to the specific target enzyme but also do so without impeding the catalytic behavior of closely related enzymes. In Figure 4 of our review we have already reported on the alignment studies to elucidate the regiospecificity of PTP1B (Scior et al 2005). The design of small molecule PTP1B inhibitors to treat type II diabetes has received considerable attention (Groves et al 1998; Wang et al 2000; Shen et al 2001; Doman et al 2002; Patankar and Jurs 2003; Sun et al 2003; Szczepankiewicz et al 2003). Vanadium compounds have been known to mimic the effects of insulin by inhibition of tyrosine phosphatases. Based on such observations that vanadate (oxo complexes of vanadium with oxidation state $+\mathrm{V}$ ) electronically and structurally imitates phosphate, and phosphorylated proteins constitute the substrates of PTP-1B, vanadium-coordinated compounds should be capable of blocking PTP-1B upon competitive replacement of the natural substrates. This is one of the possible hypotheses of the reaction mechanisms for vanadium compounds with insulin-like activities (Scior et al 2005).
In addition, more recent papers explore these mechanisms in further detail (Zborowski et al 2005; Adachi et al 2006). The discovery of the insulin-like activity (insulin-mimetics) of vanadate $\left(\left[\mathrm{VO}_{4}\right]^{3-}\right.$ or $\left.\left[\mathrm{H}_{2} \mathrm{VO}_{4}\right]^{-}\right)$(Shechter and Karlish 1980), has ushered in a new era of antidiabetes research with new oxovanadium coordination complexes with inhibitory activities against protein-tyrosine phosphatases (PTP-1B) (Huyer et al 1997), albeit not selectively. The next generation compounds have been based on organic vanadium complexes in search of higher target selectivity or less toxicity. However, some vanadium compounds have a great plethora of intrinsic enzyme affinities because of their phosphate-like physicochemical behavior: bio-isosterism (Scior et al 2005). Hence, it is of paramount importance whether the insulinmimetic effect can be dissociated from the unwanted side effects based on phosphate imitation (Scior et al 2005). The insulin-mimetic activity of certain vanadium coordination compounds has been well documented (Thompson and Orvig 2000), in particular, the improved glucose uptake and biosynthesis of glycogen as well as the inhibition of gluconeogenesis or lipolysis (Shechter et al 1998). In addition, specific biomolecular targets regarding the aforementioned effects have been identified as protein phosphatases (Posner et al 1994; Crans and Tracey 1998; Cuncic et al 1999; Tracey 2000), whereas neither insulin receptor nor GLUT2/GLUT4 pathways are directly concerned as targets (Goldwaser et al 2000). In particular, Bis-Maltolato-OxoVanadium(IV) (BMOV or $[\mathrm{VO}(\mathrm{ma}) 2]$ with central atom $=\mathrm{V} 4+$; and $\mathrm{ma}=$ monoanion maltolate) has one of the highest antidiabetic activities among the known insulin-mimetics with vanadium coordination. Especially, BMOV was used to improve insulin sensitivity (Truett 2006). Syntheses in nonaqueous solvents always yield trans-BMOV, while in water trans-and cis-aquo forms of $\mathrm{BMOV}$ are present due to interconversion (Figure 1).

Both forms have to be tested in bio-assays for their biological relevance. Hence, the exact molecular mechanism of BMOV still remains unknown (Liboiron 2005). The work also aims at studying the rotational barrier with its transition state because so far, no crystal structure of BMOV bound to PTP-1B has been reported (PDB last visited August 8th, 2008). Intriguingly, in 2003 a report presents the binding of a trigonal-bipyramidal vanadium oxide to the active site of PTP-1B although BMOV was incubated with the enzyme at the beginning of the crystallization (Peters et al 2003). Thus, whether BMOV would constitute a sort of prodrug (with an inorganic oxovanadium complex as its bioactive form) or BMOV itself might reach the binding cleft (Katherine et al 2006), constitutes the scientific motivation 


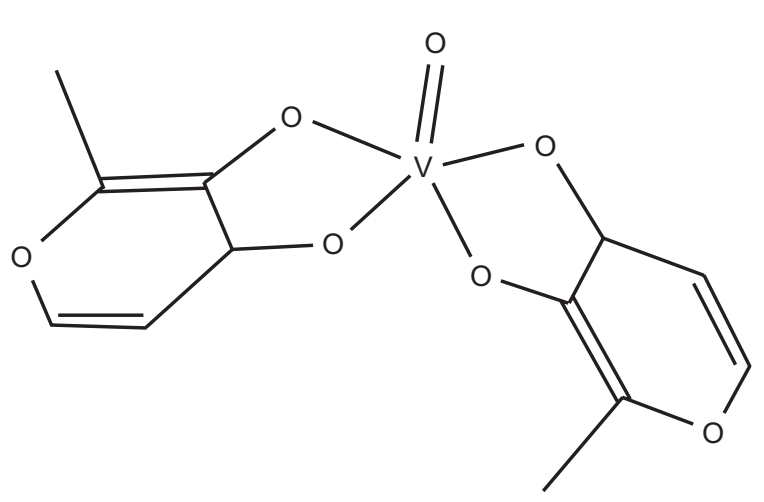

TRANS-BMOV

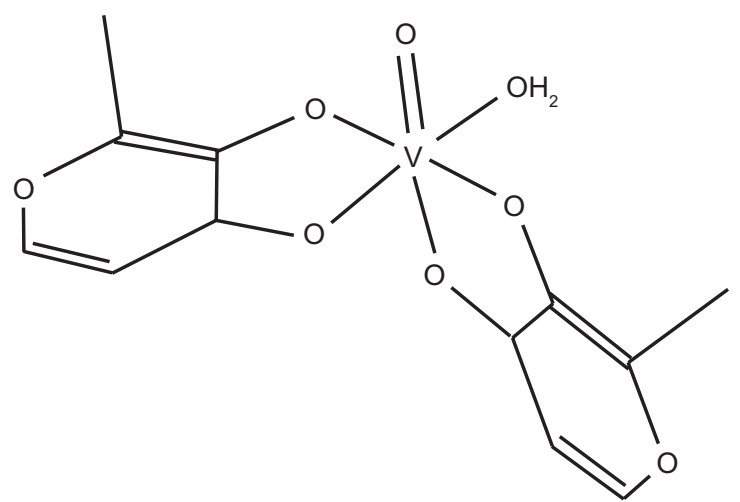

CIS-ACUO-BMOV

Figure I Structural representation of both isomers of BMOV in solution: (left) trans-BMOV, (right) the cis-acuo species where a solvent water moiety is attached to the central metal atom in the place of the electron lone pair. Despite the formal change in coordination geometries (trigonal bipyramidal to octahedral) due to the change of ligand number from 5 to 6 the over-all structure remains unchanged.

for the present in silico simulations combining geometry prediction of the ligand and its docking into the suggested target. In this context we devised ab initio calculations to elucidate BMOV geometries and related structures.

The overall goal was to gain more insight about its possible bioactive structure by modeling an amenable binding mode of BMOV and related oxovanadium complexes applying adapted force field techniques from the literature.

\section{Material and methods}

The energy minimization and unbiased, manual docking of vanadium complex into the enzyme PTP-1B was conducted in five steps on a SGI 02 work station with 126 MB RAM (Silikon 2001). In general, vanadium compounds possess a higher diversity in their oxidation states as in their geometry. Cundari and colleagues had already demonstrated good modeling accuracy using semiempirical quantum chemistry and even molecular mechanics MM2 force fields (Cundari et al 1997, 1998). Other authors applied quantum chemical methods (Zborowski et al 2005). Since both have been used successfully, we combined them in our joint study.

(1) We adapted the available Tripos force field (FF) (Sybyl; Tripos Inc., St. Louis, MO, USA) and validated it against Cundari's test series, which also contains cis-and trans-BMOV (Table 1).

(2) The Brookhaven PDB database (Berman et al 2000) was searched for crystal structures of PTP-1B in active conformation (the "open" state) with docked ligands reflecting some structural patterns: tetraoxo-complexes or aromatic "wings" (Groves et al 1998).

(3) The atoms of the target proteins were loaded with Kollman_united pre-calculated charges (Tripos Inc.) while the charges of vanadium ligands were derived from our ab initio calculations (Gaussian 1998).

(4) The geometry optimization was tailored to model the aqueous medium in a simplified approach by simulating the dumping effects of solvent molecules on electrostatic interaction in the binding groove: distance-dependent dielectric constant $(\varepsilon=40)$; nonbonded cut off at $8 \AA$; conjugate gradient algorithm: Powell, and terminal criteria: energy change: of $0.2 \mathrm{KJ} / \mathrm{mol}$ (Tripos Inc.).

(5) The final docked poses were compared to tungstate $\left(\mathrm{WO}_{4}^{-}\right.$sodium salt) bound to human PTP-1B (Barford et al 1994).

Calculations of the cis-trans conversion were carried out on two DELL-computers (Dimension 2400, with Pentium IV $2.8 \mathrm{GHz}$ processors, $256 \mathrm{MB}$ RAM, and $40 \mathrm{~GB}$ hard discs), using the Gaussian 98 electronic structure software (Gaussian, Inc., Wallingford CT, USA), and the ChemOffice 2000 program (CambridgeSoft, Cambridge, MA, USA) to visualize the results. In order to draw electronic-density and electrostatic-potential graphics, the ArgusLab software has been used (ArgusLab 2005). Together with the ChemOffice program, both BMOV structures have been generated at the B3LYP (Becke 1993) DFT level, choosing a quadratic convergence criterion ( $\mathrm{SCF}=\mathrm{QC})$. With these initial geometries, STO-3G single-point energy calculations have been performed, yielding some reference parameters of both species. Subsequently, a stepwise optimization of both chemical structures has been carried out by using the following basis sets: STO-3G, 3-21G, 3-21G*, and 6-31G. Having terminated the optimization process, frequency calculations for each structure characterized the corresponding energy minimum type (ie, a global, local or intermediate minimum). Considering the biological 
Table I Geometrical quality of the models generated under our adapted Tripos force field as compared to the reference structures published by Cundari and colleagues (1997). The geometrical similarity of the superimposed complexes is expressed as the root means square of sum for all distance differences between equivalent atoms (model vs reference) divided by the number of atoms (RMSD). RMSD itself can be seen as a descriptor of geometrical errors against a reference structure (template). If the RMSD lies under 0.2 Angstroms the small molecule is practically identical to its original template structure.

\begin{tabular}{|c|c|}
\hline Vanadium complex & RMSD [Angstroms] (comments) \\
\hline cis-BMOV & 0.18 \\
\hline trans-syn-BMOV & 0.13 \\
\hline$\left[\mathrm{V}^{+4} \mathrm{O}(\mathrm{acac})_{2}\right]$ & 0.13 \\
\hline$\left.\left[\mathrm{V}^{+4} \mathrm{O} \text { (acen) }\right)_{2}\right]$ cis & $\begin{array}{l}\text { I.07 (with lone pair electrons as formal ligands some atoms become twisted } \\
\text { and are "optimized" into a new orthohexagonal geometry due to the higher } \\
\text { coordination number with formal LPs predefinedV-coordination geometries) } \\
0.35 \text { (without formal lone pair electrons attached to V) }\end{array}$ \\
\hline$\left[\mathrm{V}^{+4} \mathrm{O}(\text { acen })_{2}\right]$ trans & 0.07 \\
\hline$\left[\mathrm{V}^{+4} \mathrm{O}(\text { catecholate })_{2}\right]^{2-}$ & 0.07 \\
\hline$\left[\mathrm{V}^{+4} \mathrm{O}\left(\mathrm{SCH}_{2} \mathrm{CH}_{2} \mathrm{~S}\right)_{2}\right]^{2-}$ crossed & 0.12 \\
\hline$\left[\mathrm{V}^{+4} \mathrm{O}\left(\mathrm{SCH}_{2} \mathrm{CH}_{2} \mathrm{~S}\right)_{2}\right]^{2-}$ parallel & 0.11 \\
\hline$\left[\mathrm{V}^{+5}(\mathrm{O})_{2}(\mathrm{dpc})\right]^{-}(\mathrm{DPOV})$ & 0.08 \\
\hline$\left[\mathrm{V}^{+5}(\mathrm{O})_{2}(\mathrm{~F})(\text { oxalate })\right]^{2-}$ & 0.10 \\
\hline$\left[\mathrm{V}^{+4}(\mathrm{O})(\mathrm{dpc})\left(\mathrm{H}_{2} \mathrm{O}\right)_{2}\right]$ & 0.12 \\
\hline$\left[\mathrm{V}^{+4}(\mathrm{O})\left(\mathrm{H}_{2} 0\right) 5\right]^{2+}$ & 0.12 \\
\hline$\left[\mathrm{V}^{+5}(\mathrm{O})_{2}(\mathrm{ox})_{2}\right]^{3-}$ cis & $\begin{array}{l}\text { I.23 (formal up-down turn of coordinated atoms, ie,the atoms change } \\
\text { position swinging through the mirror plane. No LP correction possible, cf. cis } \\
\text { form of } V+4 O(\text { acen } 2] \text { ) }\end{array}$ \\
\hline$\left[\mathrm{V}^{+5}(\mathrm{O})_{2}(\mathrm{ox})_{2}\right]^{3-}$ trans & 0.17 \\
\hline
\end{tabular}

activity of BMOV, aqueous solvent effects were taken into account. Therefore, we employed the Gaussian 98 dipole and polarization options.

In addition, DFT calculations using the B3LYP/6-311G* approach (Gaussian 03 program system; Gaussian Inc.) were carried out for trans- and cis-BMOV as well as for the transition state connecting the both isomers, in order to determine the relative stabilities of these structures.

\section{Results and discussion}

To postulate a viable mechanism one has to cope with a principle obstacle: while cis-aquo-BMOV is the observed active species, the isomeric trans-BMOV is its administered solid form. The first part of our study simulates the blocking of PTP-1B by either cis-or trans-BMOV. The calculated active configuration (cis or trans) is thought to suppress the intracellular insulin effects, therein assisting the cells in need of an adequate insulin response.

\section{Docking results}

To avoid blind docking, we conducted a PDB search for the most related crystal structure to BMOV and found a tetraoxo analogue to vanadate, called tungstate. It binds to the active site of the protein tyrosine phosphatase1B (PDB code: 2hnq) (Figure 2).

Applying the adapted Tripos FF (Tripos Inc.), the geometric structures of the BMOV and of some related complexes (Cundari et al 1997, 1998) were predicted correctly (Table 1). Prior to the unbiased docking of BMOV into the PTP-1B targets (PDB codes: 1bzc, 1bzh) (Groves et al 1998), the ligands were removed. In good agreement with reported data, trans-BMOV meets steric hindrance whatever the docked poses around the central atom position. But for the central atom of cis-BMOV, the computed pose is very similar to that described for tungstate. The final coordinates of the central atom on BMOV and of inorganic vanadate resulted in practically identical coordinates as compared to the crystallographic position of tungstate (PDB code: 2hnq) (Barford et al 1994): $\mathrm{W}$ in $\left[\mathrm{WO}_{4}\right]^{-}: \mathrm{x}=-1.1596, \mathrm{y}=-0.0977$, $\mathrm{z}=+9.2017 \mathrm{cf} . \mathrm{V}$ in $\left[\mathrm{VO}_{4}\right]^{3-}: \mathrm{x}=-1.5147, \mathrm{y}=-0.8434$, $\mathrm{z}=+10.3426$. For validation purpose, tungstate was successfully docked back into its crystallographic position using the same force field conditions (cf. $-220 \mathrm{KJ} / \mathrm{mol}$ for $\left[\mathrm{WO}_{4}\right]^{-}$). This successful validation of the test system 


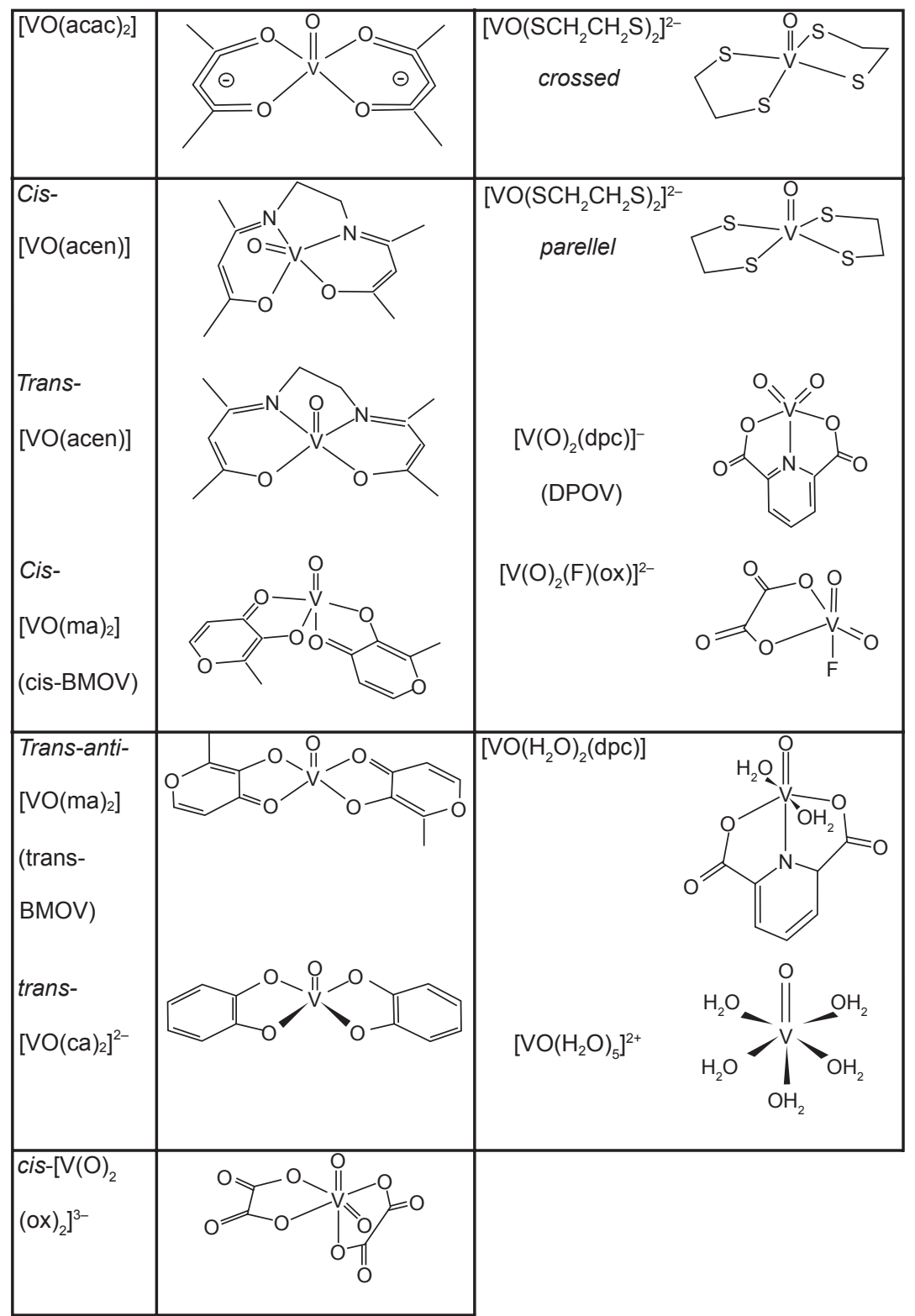

Figure 2 Structural display of reference crystal structure of protein tyrosine phosphatase IB (PDB code: $2 \mathrm{hnq}$ ). Tungstate, a tetraoxo homologue to vanadate, is bound to the active site (see text). Except for a few labeled amino acids the protein is represented by its backbone with helical parts in red, turns and loops in white, and beta strands in light blue color.

evidences that steric and electrostatic forces mainly control this anion binding. It is noteworthy that hydrophobic and solution effects (entropy) are not quantified during manual docking in the Tripos FF. Notwithstanding, the nonbonded electrostatic and van der Waals terms were combined in a qualitative manner with the insight obtained from other docking tools (Ludi, FlexX, Autodock) (Coupez and Lewis 2006). For instance, in Autodock with it's A(romatic) atom type and solvation descriptors for hydrophobic effects, $\pi-\pi$ and cation- $\pi$ interactions of neutral BMOV were recognized even without a set of vanadium parameters at hand
(Castellano et al 2003). The calculated energy (interpreted with precaution for its limited parameterization for bonded and nonbonded electrostatic/steric terms) for docked cis-BMOV was $-136 \mathrm{KJ} / \mathrm{mol}$, which lies in good proximity to the peptide ligands found in 1bzc and 1bzh after crystal strain was released by full geometry optimization (Groves et al 1998): $-179 \mathrm{KJ} / \mathrm{mol}$ and $-203 \mathrm{KJ} / \mathrm{mol}$, respectively. The bioactivity pattern of BMOV is a hybrid: on the one side, it has aromatic contacts in common with the peptide ligands, on the other side it establishes the typical hydrogen bon network of other nonpeptide small ligand complexes 
through the many oxo groups (Figure 3). Figure 8 is a schematic drawing of the binding-relevant amino acids (pharmacophore): TYR46, PHE182, SER216, ALA217, GLY218, ILE219, GLY220, and ARG221. Particularly, the residues SER216, ALA217, GLY218, ILE 219, GLY220, and cationic ARG221 participate in binding of $\left[\mathrm{WO}_{4}\right]^{-}$as an inorganic tetraoxo-monoanion whereas some of them (SER216, ALA217, GLY220, ARG221) and others (anionic ASP181, aromatic PHE182 and TYR46) interact with formally uncharged, aromatic cis-BMOV. Moreover, a $\pi-\pi$ interaction can be observed between one maltolate ring on BMOV with TYR46 (Figure 3 and 8). In addition, positively charged ARG221 attracts the other maltolate anion in a typical cation- $\pi$ interaction (Castellano et al 2003). Since both identical maltolate rings on docked BMOV enhance binding strength, but in two different ways this "wing"-symmetry can be broken up in future drug profiling in search of higher binding selectivity. In this respect, we had already aligned the PTP-1B against other phosphatase family members and have reported the results of conserved and unique amino acids at the binding site elsewhere (Scior et al 2005). As a final step in docking to confirm our working hypothesis, cis- and trans-BMOV were docked into PTP-1B. There is computed evidence that cis-aquo-BMOV can be favorably placed into the active site of the target enzyme, while transBMOV cannot enter there due to energetically high barriers (Figure 3).
Due to the absence of imaginary frequencies, the $a b$ initio optimization process for trans-BMOV yielded a global minimum (Figure 4) on the potential energy surface. Moreover, comparing both optimization results with and without solvent effects, respectively, we observed only marginal deviations. Thus, we consider this structure to be stable.

With respect to the cis-aquo-BMOV geometry, generated by stepwise single-point energy optimization, we obtained two structures: The first one, termed "closed-type cis-aquo-BMOV" (Figure 5), maintains the V-O(maltol) bonding. Theoretically, this structure represents a local minimum, due to its three imaginary frequencies, which might stem from a geometrical distortion generated by the water ligand; taking into consideration the solvent effect, this structure remains stable, too. The vacuum structure, however, seems to be slightly preferred.

The second structure (the stepwise optimization result using the 6-31G basis set) is the so-called "open-type cis-aquo-BMOV" (Figure 6). Showing the same energy as "closed-type cis-aquo-BMOV", this structure represents a global minimum, since no imaginary frequencies occurred. The solvent interaction option confirms that "open-type cis-aquo-BMOV" can be considered being stable throughout, with a slight tendency towards the aqueous phase.

Energy values obtained for the three species indicate, that in aqueous solution and room temperature (ie, under physiological conditions) the conversion from trans-BMOV

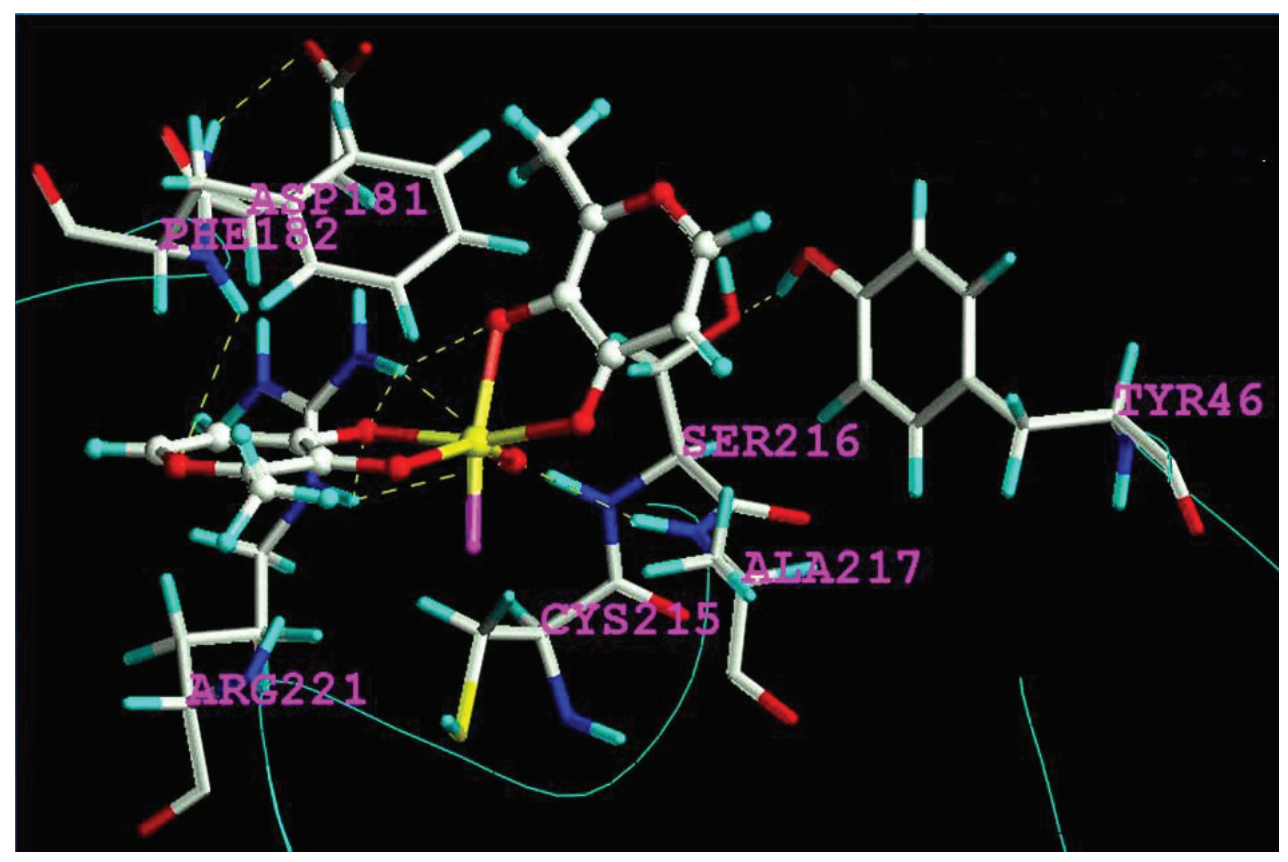

Figure 3 Result of the computed binding mode of the "closed-type cis-aquo-BMOV" complex at the active site (stick representation of 3D-model). Element color code: white $\mathrm{C}$, light blue $\mathrm{H}$, red $\mathrm{O}$, dark blue $\mathrm{N}$, yellow $\mathrm{S}$ or V, magenta LP. 


\begin{tabular}{|l|c|c|c|}
\hline BASE & OPT & DIPOLE & PCM \\
\hline STO-3G & $-1911.2523053^{*}$ & & \\
\hline STO-3G & -1911.3966987 & -1911.2325419 & -1911.2314114 \\
\hline 3-21G & -1924.1499732 & -1924.1501056 & -1924.1469983 \\
\hline 3-21G* & -1924.1499732 & -1924.1501056 & -1924.1469983 \\
\hline 6-31G & -1933.6927583 & -1933.6959770 & -1933.6924618 \\
\hline
\end{tabular}

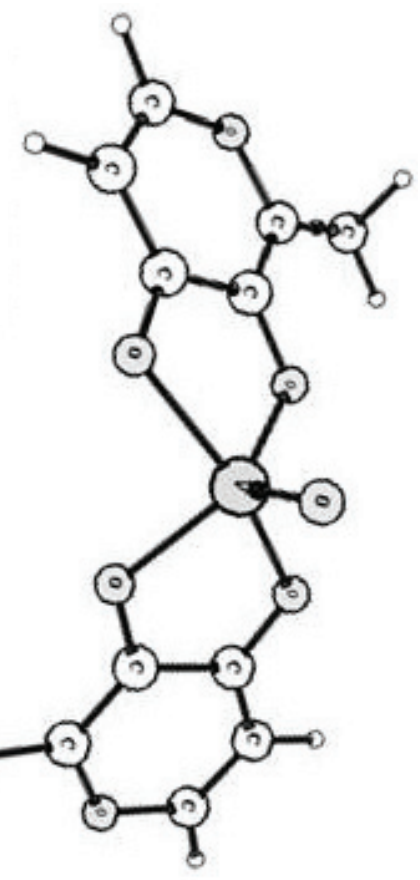

OPT $\square$ DIP $\square$ PCM

Figure 4 Results of the full optimization for the trans-BMOV complex: (Top left) Tabular listing of total energies (in Hartree) for different basis sets. The asterisk marks a local energy value. (Plot below) Total energy variation graphics (in Hartree): black: without solvent; red: with solvent; blue: interaction model with a PCM-type solvent. (Right side) Schematic plot of the optimized structure.

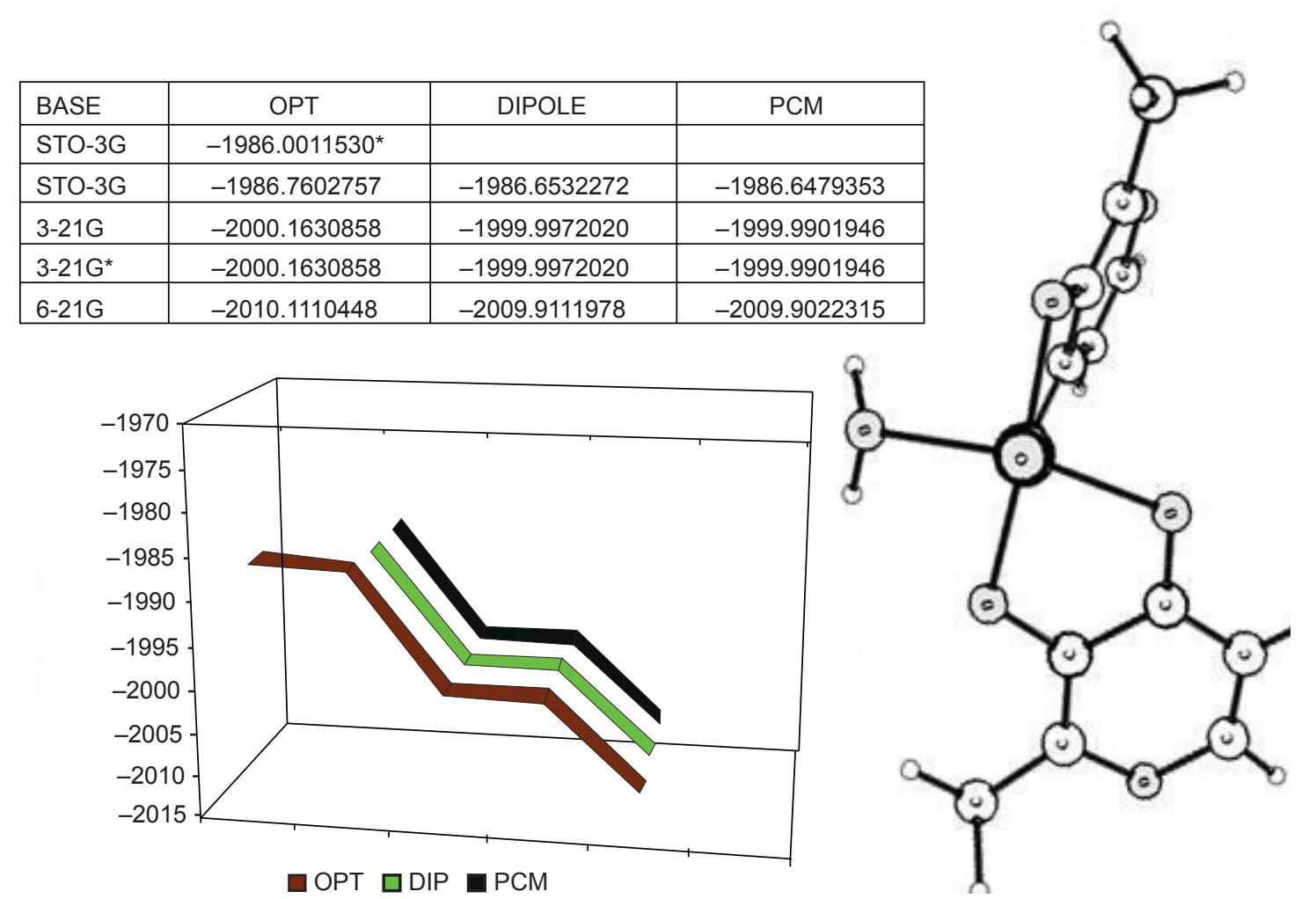

Figure 5 Results of the full optimization for the "closed-type cis-aquo-BMOV" complex: (Top left) Total energies (in Hartree) listed for different basis sets. The asterisk marks a local energy value. (Plot below) Total energy variation graphics (in Hartree): indigo blue: without solvent; green: interaction model with a dipolar solvent; blue: interaction model with a PCM-type solvent. (Right side) Schematic plot of the optimized structure. 


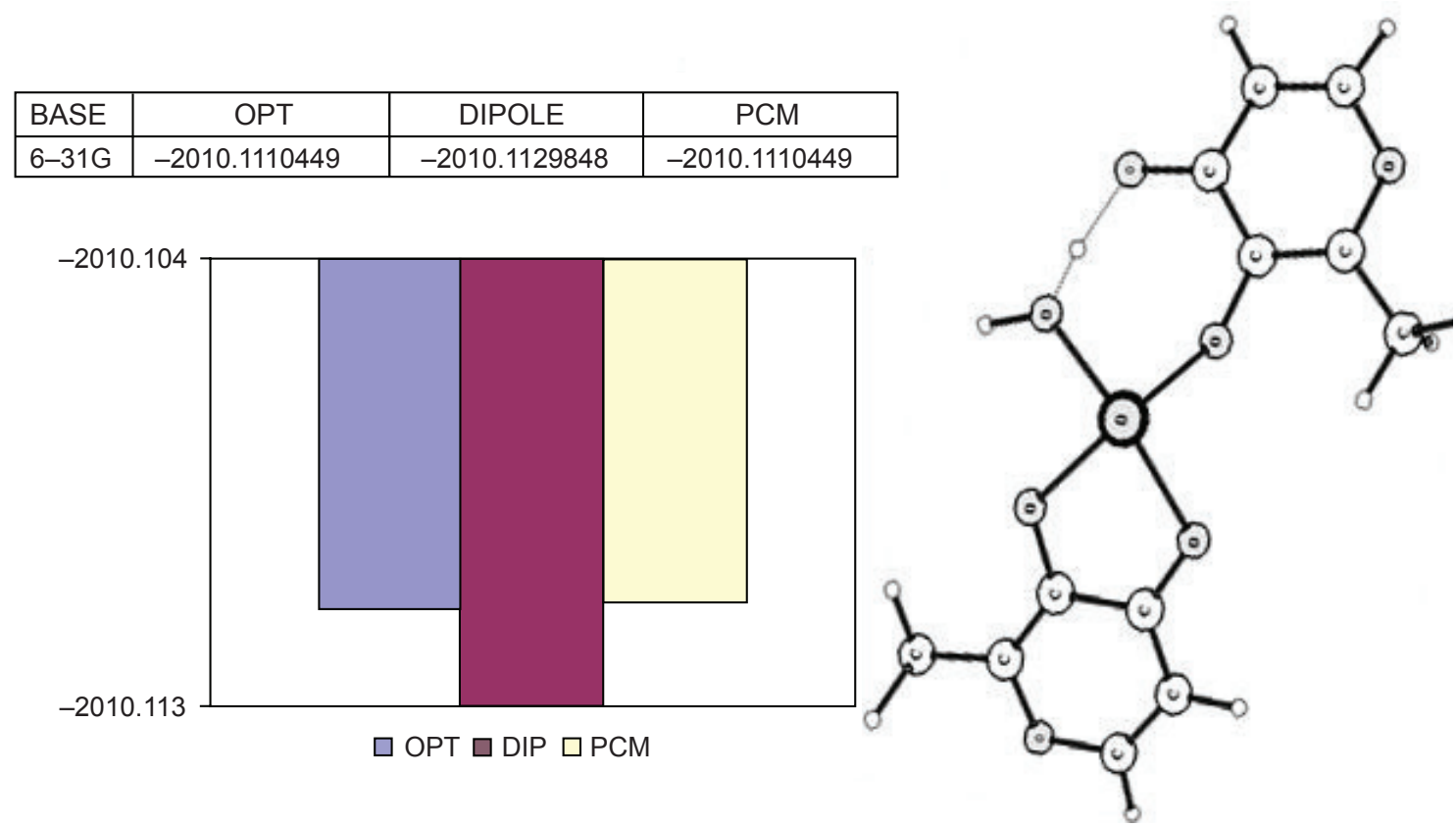

Figure 6 Results of the full optimization for the "open-type cis-aquo-BMOV” complex: (Top left) Total energies (in Hartree) for the basis set 6-3। G. (Plot below) Total energy bar graphics (in Hartree): blue: without solvent; red: interaction model with a dipolar solvent; yellow: interaction model with a PCM-type solvent. (Right side) Schematic plot of the optimized structure.

to cis-aquo-BMOV is thermodynamically possible. The "open-type cis-aquo-BMOV" hereby seems to be the stable intermediate in kinetic equilibrium with the "closed-type cis-aquo-BMOV" form. To find out which of the two species interacts with the enzyme we determined both corresponding electrostatic potential surfaces by means of the Arguslab program (ArgusLab 2005). The result confirms that "closedtype cis-aquo-BMOV" shows a negative electrostatic potential of higher absolute value at the vanadium position and its close environment (Figure 7). It thus turns out to be more nucleophilic compared to the "open" structure, with higher affinity to the catalytic center of the enzyme PTP-1B.

Finally, the geometries of trans- and cis-aquo-BMOV ("open-type") were optimized at the B3LYP/6-311G* level of theory. Subsequent frequency calculations demonstrated, that both forms represent minima, ie, stable structures, on the energy hypersurface. The cis isomer is lower in energy by $5.6 \mathrm{KJ} / \mathrm{mol}$ as compared with the trans-BMOV, thus being the global minimum. The search for a transition state connecting the both forms along the reaction coordinate resulted in a structure possessing one imaginary frequency, ie, a first order saddle point, being higher in energy by $21.1 \mathrm{KJ} / \mathrm{mol}$ with respect to the global minimum.

\section{Conclusion}

The goal of the present in silico study is to theoretically address the missing explanation of a trans-cis conversion of BMOV.
To our best knowledge, it generates the first molecular binding mode model concerning the insulin-mimetic activity of BMOV. It also elucidates the stereochemical requirements of BMOV as an inhibitor to the biomolecular target site. In aqueous solution the administered compound transBMOV is converted into an "open-type cis-aquo" species of BMOV, which itself is in equilibrium with another "closed-type" form of cis-aquo-BMOV. The postulated binding mode for BMOV, ie,"closed-type cis-aquo-BMOV" complex, is fully consistent with in vivo studies on BMOV activity with respect to enzyme activity of PTP-1B: the group of $\mathrm{McNeill}$ reported that BMOV treatment reduced the activity of PTP I B by $25 \%$ in fatty treated rats (Mohammad et al 2002).

Our present work may lead to the detection of the activated complex upon forming a complex of cis-BMOV with a $\mathrm{H}_{2} \mathrm{O}$ moiety. It defines the energy barrier, which in turn may control the reaction speed. Our report may help other researchers to devise new calculations concerning this activated complex. To this end our modeling input and output data can be obtained by email.

In conclusion, we contribute with computed solutions to better understand the molecular recognition process and selectivity of nonpeptide insulin-mimetic vanadium complexes. To complete the picture, on a theoretical level it cannot be discarded that vanadium coordinated organic 


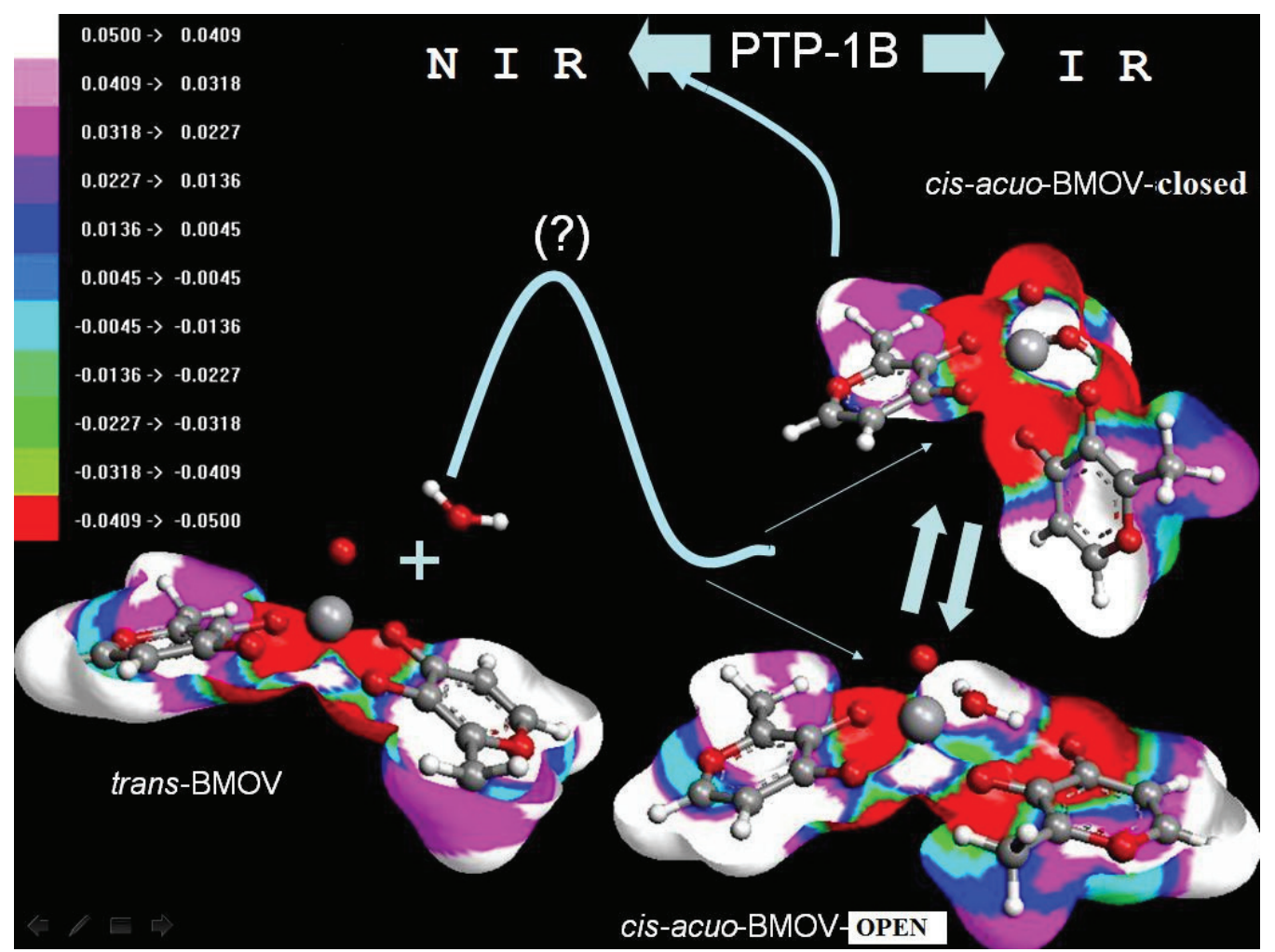

Figure 7 Energy diagram of the computed cis-trans conversion mechanism for the bioactive BMOV in its "closed-type cis-aquo"-form. In aqueous solution, the administered compound, trans-BMOV, intermediately converts into the "open-type cis-aquo-BMOV" complex being in equilibrium with the "closed-type cis-aquo-BMOV". Note, the activated transition complex of the reaction trans-BMOV $+\mathrm{H}_{2} \mathrm{O}$ remains hitherto unknown. NIR, normal insulin response; IR, state of insulin resistance.

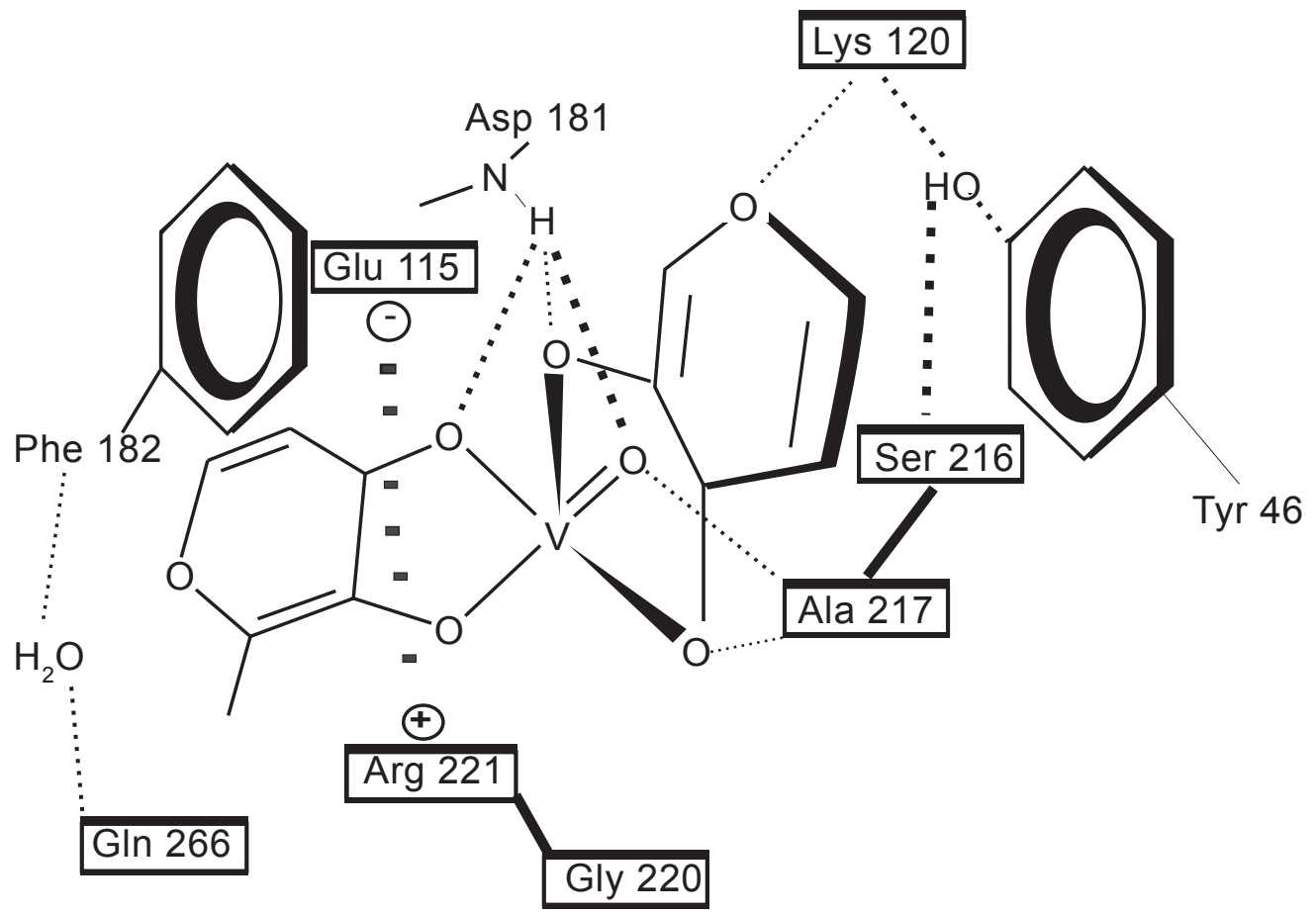

Figure 8 Schematic viewing of our elaborated pharmacophore model with the active site of PTP-IB: binding- relevant amino acids are labeled according to the numbering scheme found in PDB entry: 2hnq. Not shown: upon blocking the enzyme PTP-IB, the insulin docked to its extracellular membrane receptor triggers the intercellular signal and the cell responds with normal glucose up-take. Boxed amino acids are not structurally depicted just labeled by their code for figure clarity. 
compounds would also be prodrugs after re-arrangements with stronger chelators and following redox-reactions. Thus, whether BMOV or a simple (inorganic) tetraoxo compound is the final bioactive species acting on target structure remains an open question and disserves further investigation.

\section{Acknowledgments}

For their pancreatic and extra-pancreatic exploration of our synthetic vanadium complexes special thanks are owed to Priv. Doz. Dr Nicolas Lembert, and Priv. Doz. Dr Reiner Lammers, at University of Tuebingen, Germany. For temporal work of TS and JAG at University of Tuebingen, Germany, and at GreenPharma, Orléans, France, grateful acknowledgments are given to Prof. Dr. S. Laufer, Dr D. Domeyer. Dr Q.-T. Do, and Dr Ph. Bernard. We also would like to acknowledge Dr P.H. Hernandez T. of VIEP - BUAP, as well as CONACYT (2007/52639) for their valuable support.

\section{References}

Adachi Y, Yoshikawa Y, Yoshida J, et al. 2006. Improvement of diabetes, obesity and hypertension in type 2 diabetic $\mathrm{KKA}(\mathrm{y})$ mice by bis(alli-xinato)oxovanadium(IV) complex. Biochem Biophys Res Commun, 345:945-50.

ArgusLab. 2005. ArgusLab 4.0.1. Mark A. Thompson. Planaria Software LLC, Seattle, WA.

Atkins PW, Friedman RS. 2003. Molecular Quantum Mechanics. Oxford University Press. Oxford, New York, Toronto.

Aznar CP, Deligiannakis Y, Tolis EJ, et al. 2004. ESE-ENDOR study and DFT calculations on oxovanadium compounds: Effect of axial anionic ligands on the ${ }_{51}$ V nuclear quadrupolar coupling constant. J Phys Chem, 108:4310-21.

Barford D, Flint AJ, Tonks NK. 1994. Crystal structure of human protein tyrosine phosphatase 1B. Science, 263(5152):1397-404.

Becke AD. 1993. Density-functional thermochemistry. III. The role of exact exchange. J Chem Phys, 98:5648-52.

Bella SD, Lanza G, Gulino A, et al. 1996. Electronic structure of Bis (2, 4-pentanedionato-O; O') oxovanaduim (IV). A photoelectron spectroscopy, electronic spectroscopy, and ab initio molecular orbital study. Inorg Chem, 35:3885-90.

Berman HM, Westbrook J, Feng Z, et al. 2000. The protein data bank. Nucl Acid Res, 28:235-42.

Bose S, Farah MA, Jung HC, et al. 2007. Molecular mechanism of bis(mal tolato)oxovanadium(IV)-induced insulin signaling in 3T3-L1 and IM9 cells: impact of dexamethasone. J Mol Endocrinol, 38:627-49.

Bühl M. 2000. Density-functional study of vanadate-glycylserine isomers. J Inorg Biochem, 80:137-9.

Caballero CNA. 2003. Master Thesis in Chemical Sciences in Spanish language: Tesis de Maestría en Ciencias Químicas. Estudio Teórico de Compuestos de Vanadio con Actividad Tipo Insulina y su Interacción con la Enzima PTP-1B. Benemérita Universidad Autónoma de Puebla. Puebla, México.

Castellano RK, Diederich F, Meyer EA. 2003. Interactions with Arenes: Interactions with Aromatic Rings in Chemical and Biological Recognition. Angew Chem, 42:1210-40.

Coupez B, Lewis RA. 2006. Docking and scoring - theoretically easy, practically impossible? Curr Med Chem, 13:2995-3003.

Crans DC. 2000. Chemistry and insulin-like properties of vanadium(IV) and vanadium(V) compounds. J Inorg Biochem, 80:123-31.
Crans DC, Tracey AS, Crans DC. 1998. Vanadium Compounds: Chemistry, Biochemistry, and Therapeutic Applications. Washington, DC: American Chemical Society Symposium Series.

Cuncic C, Detich N, Ethier D, et al. 1999. Vanadate inhibition of protein tyrosine phosphatases in Jurkat cells: modulation by redox state. $J$ Biol Inorg Chem. 4:354-9.

Cundari TR, Saunders L, Sisterhen LL, 1998. Molecular modeling of vanadium oxo complexes. a comparison of quantum and classical methods. J Phys Chem A, 102:997-1004.

Cundari TR, Sisterhen LL, Stylianopoulos CL, 1997. Molecular modeling of vanadium peroxides. Inorg Chem, 36:4029-34.

Cheng A, Dubé N, Gu F, et al. 2002. Coordinated action of protein tyrosine phosphatases in insulin signal traduction. Eur J Biochem, 269:1050-9.

Doman TN, McGovern SL, Witherbee BJ, et al. 2002. Molecular docking and high-throughput screening for novel inhitors of protein tyrosine phosphatase-1B. J Med Chem, 45:2213-21.

Elchebly M, Payette P, Michaliszyn E, et al. 1999. Increased insulin sensitivity and obesity resistance in mice lacking the protein tyrosine phosphatase-1B gene. Science, 283:1544-8.

Foresman JB, Frisch AE. 1998. Exploring Chemistry with Electronic Structure Methods: A Guide to Using Gaussian 98. Wallingford, CT: Gaussian, Inc.

Gracia L, Andrés J, Safont VS, et al. 2004. DFT study of the reaction between $\mathrm{VO} 2+$ and C2H6. Organometallics, 23:730-9.

Groves MR, Yao ZJ, Roller PP, et al. 1998. Structural basis for inhibition of the protein tyrosine phosphatase $1 \mathrm{~B}$ by phosphotyrosine peptide mimetics, Biochemistry, 37:17773-83.

Goldwaser I, Gefel D, Gershonov E, et al. 2000. Insulin-like effects of vanadium; basic and clinical implications. J Inorg Biochem. 80:21-5.

Guevara JA. 1996. Una Semblanza de la Química Bioinorgánica del Vanadio. Revista Educación Química, 7:185-9.

Heinemann G, Fichtl B, Vogt W. 2003. Pharmacokinetics of vanadium in humans after intravenous administration of a vanadium containing albumin solution. Br J Clin Pharmacol, 55:241-5.

Huyer G, Liu S, Kelly J, et al. 1997. Mechanism of inhibition of protein-tyrosine phosphatases by vanadate and pervanadate. $J$ Biol Chem, 272:843-51.

Jindal S, Singh M, Balakumar P. 2007. Effect of bis (maltolato) oxovanadium (BMOV) in uric acid and sodium arsenite-induced vascular endothelial dysfunction in rats. Int $J$ Cardiol, 1:23-7.

Kallies B, Meier R. 2001. Electronic structure of 3d [M(H2O)6]3+ ions from ScIII to FeIII: A quantum mechanical study based on DFT computations and natural bond orbital analyses. Inorg Chem, 40:3101-12.

Katherine H, Thompson, Orvig C, 2006. Metal complexes in medicinal chemistry: new vistas and challenges in drug design. Dalton Trans, (6):761-4.

Larsen SC. 2001. DFT calculations of proton hyperfine coupling constants for $[\mathrm{VO}(\mathrm{H} 2 \mathrm{O}) 5] 2+$ : Comparison with proton ENDOR data. $J$ Phys Chem, 105:8333-8.

Levine IN. 2000. Quantum Chemistry. Upper Saddle River, NJ: Prentice Hall.

Liboiron BD, Thompson KH, Hanson GR, et al. 2005. New insights into the interactions of serum proteins with Bis(maltolato)oxovanadium (IV): Transport and biotransformation of insulin-enhancing vanadium pharmaceuticals. J Am Chem Soc, 127:5104-15.

McQuarrie DA, Simon JD.1997. Physical Chemistry, a Molecular Approach. Sausalito, CA: University Science Books.

Mohammad A, Wang J, McNeill JH. 2002. Bis(maltolato)oxovanadium(IV) inhibits the activity of PTP1B in Zucker rat skeletal muscle in vivo. Mol Cell Biochem, 229:125-8.

Patankar SJ, Jurs PC. 2003. Classification of inhibitors of protein tyrosine phosphatase 1B using molecular structure based descriptors. J Chem Inf Comput Sci, 43:885-9.

Peters KG, Davis MG, Howard BW, et al. 2003. Mechanism of insulin sensitization by BMOV (bis maltolato oxo vanadium); unliganded vanadium (VO4) as the active component. J Inorg Biochem, 96:321-30.

Pople JA. 1999. Quantum chemical models (Nobel Lecture). Angew Chem, 38:1894-902. 
Posner BI, Faure R, Burgess JW, et al. 1994. Peroxovanadium compounds. A new class of potent phosphotyrosine phosphatase inhibitors which are insulin mimetics. J Biol Chem, 269:4596-604.

Saatchi K, Thompson KH, Patrick BO, et al. 2005. Coordination chemistry and insulin-enhancing behavior of vanadium complexes with maltol C6H6O3 structural isomers. Inorg Chem, 44:2689-97.

Scior JT, Guevara JA, Bernard P, et al. 2005. Are vanadium compounds drugable? Structures and effects of antidiabetic vanadium compounds: a critical review. Mini Rev Med Chem, 5:1-14.

Shechter Y, Eldberg G, Shisheva A, et al. 1998. Vanadium Compounds, Chemistry, Biochemistry and Therapeutic Applications. New York, NY: Oxford University Press.

Shechter Y, Karlish SJ. 1980. Insulin-like stimulation of glucose oxidation in rat adipocytes by vanadyl (IV) ions. Nature, 284:556-8.

Shen K, Keng YF, Wu L, et al. 2001. Acquisition of a specific and potent PTP1B inhibitor from a novel combinatorial library and screening procedure. J Biol Chem, 276:47311-9.

Sun JP, Fedorov AA, Lee SY, et al. 2003. Crystal structure of PTP1B complexed with a potent and selective bidentate inhibitor. J Biol Chem, 278:12406-14.
Szczepankiewicz BG, Liu G, Hajduk PJ, et al. 2003. Discovery of a potent, selective protein tyrosine phosphatase $1 \mathrm{~B}$ inhibitor using a linkedfragment strategy. J Am Chem Soc, 125:4087-96.

Thompson KH, Orvig C. 2000. Design of vanadium compounds as insulin enhancing agents. J Chem Soc-Dalton Trans, 17:2885-92.

Tracey AS. 2000. Hydroxamido vanadates: aqueous chemistry and function in protein tyrosine phosphatases and cell cultures. J Inorg Biochem, 80:11-6.

Truett GE. 2006. Prevention of diabetes by inhibition of tyrosine phosphatases. Exp Biol Med, 231:487-8.

Wang H, Lim KL, Yeo SL, et al. 2000. Isolation of a novel protein tyrosine phosphatase inhibitor, 2-methyl-fervenulone, and its precursors from Streptomyces. J Nat Prod, 63:1641-6.

Zhang SQ, Zhong XY, Chen GH, et al. 2008. The anti-diabetic effects and pharmacokinetic profiles of bis(maltolato)oxovan adium in non-diabetic and diabetic rats. J Pharm Pharmacol, 60:99-105.

Zborowski K, Grybos R, Proniewicz LM. 2005. Molecular structures of oxovanadium(IV) complexes with maltol and kojic acid: a quantum mechanical study. Inorg Chem Comm, 8:76-8. 
